\title{
Evaluation Of Standard Magnetic Resonance Characteristics Used To Differentiate Neoplastic, Inflammatory, And Vascular Brain Lesions In Dogs
}

Benjamin D. Young, Geoffrey T. Fosgate, Shannon P. Holmes, Collin A. Wolff, Annie V. Chen-Allen, Marc Kent, Simon R. Platt, Mason Y. Savage, Scott J. Schatzberg, Jonathan M. Levine

Key words: brain, dog, imaging, neoplasia, inflammation

Running Head: Evaluation of MR Brain Lesion Characteristics

Funding sources: none

Previous presentations or abstracts: none 
ABSTRACT

Magnetic resonance (MR) imaging has been shown to be highly sensitive and specific for identifying neoplastic and inflammatory intracranial disease in dogs. Previously seven MR characteristics were shown to distinguish neoplasia from other intracranial disease. Our goal was to identify MR characteristics or combinations of characteristics that could broadly distinguish neoplastic, inflammatory, and vascular intracranial diseases in dogs. A total of 75 dogs with histologically confirmed intracranial disease were retrospectively identified (51 with neoplasia, 18 with inflammatory disease, and 6 with cerebrovascular disease). Three blinded examiners evaluated 19 MR lesion characteristics totaling 57 possible responses. Only strong contrast enhancement was more common in neoplasia than other disease categories. A multivariable statistical model suggested that extra-axial origin, T2-FLAIR mixed intensity, and defined lesion margins were also predictive of neoplasia. Meningeal enhancement, irregular lesion shape, and multifocal location distinguished inflammatory diseases from the other disease categories. No MR characteristics distinguished vascular lesions, which appeared most similar to neoplasia. 


\section{INTRODUCTION}

Magnetic resonance (MR) is now integral to the non-invasive diagnosis of intracranial disease at many veterinary referral and university teaching hospitals. A presumptive diagnosis based on signalment, imaging, clinical findings, and cerebrospinal fluid (CSF) analysis is commonly used to establish a patient's prognosis and therapeutic plan in the absence of histologic diagnosis. A growing body of literature has described the MR characteristics of numerous intracranial diseases in the dog, most commonly in case reports and occasionally in larger retrospective case series. These studies tend to show MR findings are often non-specific and are shared between major disease categories. ${ }^{1-10}$ Familiarity with the histologic behavior of diseases may aid in MR interpretation. ${ }^{11,12}$ However histopathological lesions are not reliably reflected in MR findings, and variability of MR signal intensity or contrast enhancement can be seen with neuronal loss, vascular damage, perilesional edema, and necrosis. ${ }^{13-15}$

Because of the potential complications associated with brain biopsy, the importance of neuroimaging in disease classification is recognized in human medicine and has motivated the study of MR performance evaluation and the creation of imaging finding databases. ${ }^{16}$ Similarly in veterinary medicine, a recent multi-institutional retrospective study of 121 client-owned dogs with either histologically confirmed brain disease or idiopathic epilepsy found routine high field MR to be $94.4 \%$ sensitive and $95.5 \%$ specific for overall detection of brain lesions with very good inter-rater agreement. ${ }^{17}$ In that study the highest performance was reported for correctly categorizing neoplastic and inflammatory lesions with a lower sensitivity for cerebrovascular lesions. There was high specificity but low sensitivity for identifying many diseases suggesting variable disease appearance and limitations of MR diagnosis, similar to human studies. ${ }^{18}$ Interestingly in that study the inclusion of patient clinical data did not improve the accuracy of diagnosis, and in certain diseases (such as glioma) it actually reduced specificity and inter-rate agreement. When relying solely on imaging features, reviewers were as successful at assigning the 
correct disease category and seemingly less likely to fall victim to bias in diagnosing an underlying disease process as when subsequently provided with clinical data. ${ }^{17} \mathrm{~A}$ subsequent paper similarly found high interobserver agreement between MR and clinical diagnosis in 44 dogs. ${ }^{19}$

Imaging diagnosis requires both recognition of the MR features of tissue damage and a rubric for differentiating between brain disease categories. To the latter, the suggested MR appearance of various brain diseases presumably provides a basis for decision making. A previous study of 36 dogs and 13 cats identified $7 \mathrm{MR}$ characteristics that could be used to differentiate intracranial neoplasia from nonneoplastic disease. ${ }^{20}$ In that population, single lesion, regular shape, dural contact, dural tail, bone lesions, mass effect, and the presence of contrast enhancement were significantly more common in neoplasia than in inflammatory or vascular diseases. Importantly, the majority of neoplasias in that report were meningiomas (51.5\%), which may have affected the reported result. The imaging characteristics of many types of intra-axial neoplasia may be varied and quite different than tumors such as meningioma. ${ }^{2,3,5,21-25}$ To evaluate the repeatability of these conclusions, the aim of the study presented here was to identify MR characteristics or combinations of characteristics that differentiate the broad categories of intracranial neoplastic, inflammatory, and cerebrovascular lesions in a multiinstitutional population of dogs.

\section{MATERIALS AND METHODS}

Medical records from 2005 to 2011 were searched for dogs presenting with signs of intracranial neurologic disease at 3 veterinary medical teaching hospitals: Texas A\&M University (TAMU), the University of Georgia (UGA), and Washington State University (WSU). Search criteria were described in a previous report utilizing the same population of dogs. ${ }^{17}$ Briefly, cases of intracranial disease were required to have an antemortem brain magnetic resonance (MR) at $1 \mathrm{~T}$ or higher field strength and 
histopathological diagnosis of inflammatory (immune-mediated, infectious, or unknown etiology), neoplastic (primary or secondary), or cerebrovascular (ischemic or hemorrhagic) brain disease obtained by either biopsy or necropsy. There were 77 dogs that were identified with intracranial disease. These included 53 dogs with intracranial neoplasia, 18 dogs with inflammatory disease, and 6 dogs with cerebrovascular disease.

For study inclusion all MR studies had both transverse and sagittal spin echo (SE) or fast spin echo (FSE) T2-weighted images (T2W), transverse T2-weighted fluid attenuated inversion recovery images (T2FLAIR), and transverse pre- and post-contrast T1-weighted SE (T1W) or FLAIR (T1-FLAIR) images. Studies were acquired on either 1.0T (Magnetom Expert Plus, Siemens Medical USA, Malvern, PA, USA; Gyroscan NT Intera, Philips Medical Systems, Best, Netherlands) (T2W: repetition time (TR) 1945-4385 ms, echo time (TE) 90-120 ms, slice thickness 2-5 mm; T1W: TR 350-889 ms, TE 10-20 ms, slice thickness 2-6 mm; T2-FLAIR: TR 7500-1100 ms, TE 119-140 ms, slice thickness 3-5 mm) or a 3.0T (Signa HDx, General Electric Healthcare, Milwaukee, WI, USA) (T2W: TR 3000-4000 ms, TE 100-116 ms, slice thickness 2-3 mm; T1-FLAIR: TR 2470-2900 ms, TE 8-10 ms, slice thickness 3 mm; T2-FLAIR: TR 9502 ms, TE 120-128 ms, slice thickness $3 \mathrm{~mm}$ ) field strength systems. Intravenous gadolinium-based contrast medium (gadopentetate dimeglumine, Magnevist, Bayer Healthcare Pharmaceuticals, Wayne, NJ, USA) was used in each case at a dose of $0.1 \mathrm{mmol} / \mathrm{kg}$. The time delay between administration of contrast medium and post-contrast imaging was neither standardized between institutions nor recorded in each case. No additional image planes or sequences were made available to reviewers or included in the analysis. For dogs with multiple MR studies, only the study performed nearest the time of lesion biopsy or necropsy was included in analysis.

All studies were evaluated in digital format by three investigators, each with at least 5 years of experience in brain MR evaluation, two board-certified radiologists (B.D.Y., S.P.H.) and a board-certified 
neurologist (J.M.L.). All studies were anonymized and randomized by one investigator (C.A.W.). Data for the current study was obtained simultaneous to a previously published study. ${ }^{17}$ Evaluators were first asked whether the brain was normal or abnormal. If one or more lesions were identified, they were then asked to evaluate 19 MR characteristics of the lesions with 57 possible responses, which included: lesion number (single, multiple); lesion shape (spherical, papilliform, angular, irregular); lesion margins (defined, ill-defined); T1W, T2W, and T2-FLAIR signal uniformity (homogenous, heterogeneous); T1W, T2W, and T2-FLAIR signal intensity relative to normal gray matter (hypo-, iso-, hyperintense, mixed intensity); contrast enhancement pattern (none, homogenous, heterogeneous, rim-enhancement); contrast enhancement intensity (absent, weak (hypointense to fat), strong (isointense to fat), comparison to fat made on sequences without fat suppression); axial origin (intra-, extra-axial, ambiguous); location (supra-, infratentorial, periventricular, multifocal); mass effect (yes, no); parenchymal distribution (gray matter, white matter, mixed); meningeal contact (yes, no); meningeal enhancement (absent, leptomeningeal, pachymeningeal); dural tail sign (yes, no); osseous changes (absent, lysis, thickening). Intraventricular lesions and those identified as originating from the pituitary gland were designated as "extra-axial" in origin.

\section{STATISTICAL ANALYSIS}

Categorical MR characteristics data were summarized by disease diagnosis using frequencies, proportions, odds ratios (OR), and 95\% confidence intervals (Cl). Mixed-effects logistic regression was used to estimate the associations between MR characteristics and disease diagnosis adjusting for the repeated observations ( 3 readers per dog) using a random effect for dog. All 19 MR characteristics were first screened using a univariate analysis and characteristics with $p \leq 0.20$ were evaluated in a multivariable model. Screening models were evaluated with the MR characteristic as the dependent (outcome) variable and the predictor (independent) variable the diagnostic category of neoplasia, 
inflammatory, or vascular disease. Multivariable models were constructed for an outcome of neoplastic disease using a backwards stepwise approach based on the $t$ statistics calculated from regression coefficients. Non-significant predictors were removed one-by-one based on $p>0.05$. Interaction terms were not investigated during multivariable modeling. Univariate screening models were used for the calculation of proportions and $95 \%$ confidence intervals and performing post hoc pairwise comparisons employing Bonferroni $p$ value adjustments. Within the neoplastic group, the sub-groups meningioma, glioma, and other neoplasia were similarly analyzed. Further evaluation of inflammatory diseases was performed after categorization as granulomatous meningoencephalitis (GME), necrotizing encephalitis (NE), and other inflammatory conditions. Modeling was not performed within vascular diagnoses due to the limited number of cases. Mantel-Haenszel chi-square tests stratified by individual readers were performed when zero marginal totals prevented the mixed-effects models from estimating effects. Statistical modeling was performed using commercially available software (IBM SPSS Statistics Version 21, International Business Machines Corp., Armonk, NY, USA) and results interpreted at the $5 \%$ level of significance.

\section{RESULTS}

The magnetic resonance (MR) study from one dog with lymphoma was interpreted as "normal" by all reviewers and was removed from analysis due to lack of scoring. Another dog diagnosed with 2 neoplasia types (pituitary adenoma and unspecified glioma) was excluded because it was not possible to determine which disease was primary. Therefore there were a total of 75 dogs included in the study analysis: 51 with neoplasia, 18 with inflammatory disease, and 6 with cerebrovascular disease. The median age for dogs with neoplasia was 9 years (range 3-14 years), for dogs with inflammatory disease was 5 years (range 1-10 years), and for dogs with vascular disease was 11 years (range 1-13 years). 
Among dogs with neoplasia, 2 were intact males, 27 were castrated males, 1 was an intact female, and 21 were spayed females. Among dogs with inflammatory disease, 2 were intact males, 8 were castrated males, 3 were intact females, and 5 were spayed female. Among dogs with vascular disease, 2 were intact males, 2 were castrated males, 1 was an intact female, and 1 was a spayed female. Among all disease categories, breeds included: Golden Retriever (9), Boxer (7), mixed breed (7), Labrador Retriever (6), Boston Terrier (5), American Staffordshire Terrier (3), Australian Shepherd (3), Bulldog (3), Pug (3), and 24 other breeds with $\leq 2$ dogs each. Histopathological diagnoses for all neoplasias included: meningioma (19), glioma [oligodendroglioma (11); astrocytoma (2); unspecified (1); mixed (1)], pituitary adenocarcinoma/carcinoma (4), choroid plexus carcinoma (3), invasive nasal adenocarcinoma (2), lymphoma (1), nerve sheath tumor [ganglionneuroma (1); perineurioma (1)], ependymoma (1), hemangiosarcoma (1), medulloblastoma (1), metastatic apocrine gland anal sac adenocarcinoma (1), and multilobulated bone tumor (1). Histopathological diagnoses for all inflammatory diseases included: granulomatous meningoencephalitis (GME) (8), necrotizing encephalitis (NE) [necrotizing meningoencephalitis (NME) (4); necrotizing leukoencephalitis (NLE) (1)], meningoencephalitis of unknown etiology (MUE) (3), and 2 cases of infectious meningoencephalitis [fungal (1); bacterial (1)]. Histopathological diagnoses for all vascular diseases included: hemorrhagic infarct (3) and ischemic infarct (3). The median time from onset of clinical signs to MR exam was 34 days (range, 1-280 days) for neoplasia, 5.5 days (range, 0.5-90 days) for inflammatory disease, and 3.5 days (range, 1-14 days) for vascular disease.

A comparison of MR characteristics between major disease categories for results in which $p<0.20$ is presented in Table 1. There was only one characteristic that was more common in neoplasia than either inflammatory or vascular diseases: strong contrast enhancement $(p=0.003)$. There was only 1 characteristic that was less common in neoplasia than either inflammatory or vascular diseases: intraaxial origin $(p=0.002)$. There were 3 characteristics that were more common for inflammatory disease 
than either neoplasia or vascular diseases: meningeal enhancement $(p=0.003)$, irregular lesion shape ( $p$ $<0.001)$, and multifocal location $(p<0.001)$. Defined lesion margins $(\mathrm{OR}=11.9 ; 95 \% \mathrm{Cl} 2.54-55.3), \mathrm{T} 2-$ FLAIR mixed intensity $(\mathrm{OR}=7.5095 \% \mathrm{Cl} 1.55-36.4)$, and extra-axial lesion origin (OR $=14.395 \% \mathrm{Cl} 2.17$ 93.9) were independent predictors of neoplastic lesions compared to the inflammatory and vascular groups combined (Table 2).

For dogs with diagnosis of intracranial neoplasia, a comparison of MR characteristics was performed for the most common tumor types (meningioma and glioma) against all other neoplasias (Table 3). Despite the presence of significant associations in the univariate analysis, when MR characteristics were combined in a multivariable model there was no combination that was able to differentiate meningiomas from the other tumor types. Intra-axial origin was the only variable more common to glioma than all other tumor types $(p<0.001)$.

For dogs with diagnosis of intracranial inflammatory disease, intra-axial origin $(p=0.184)$ and supratentorial location $(p=0.106)$ were identified as being potentially important for differentiating GME and NE from other inflammatory diseases in the univariate screening models but were not significant predictors when combined in a multivariable model. The proportion $(95 \% \mathrm{Cl})$ of dogs with a lesion of intra-axial origin was $0.97(0.66,1.00), 1.00(0.00,1.00)$, and $0.52(0.10,0.92)$ for $\mathrm{GME}, \mathrm{NE}$, and other diseases, respectively. The proportion $(95 \% \mathrm{Cl})$ of dogs with a supratentorial lesion was $0.12(0.02$, $0.47), 0.70(0.23,0.95)$, and $0.51(0.15,0.87)$ for $\mathrm{GME}, \mathrm{NE}$, and other diseases, respectively.

\section{DISCUSSION}

In the population of 75 dogs with histologically diagnosed brain lesions reported here, there were only two magnetic resonance (MR) characteristics that distinguished neoplasia from other disease 
categories: strong contrast enhancement was more common with neoplasia and intra-axial origin was less common. However, the multivariable model also suggested that extra-axial origin, T2-FLAIR mixed intensity, and defined lesion margins were independent predictors of neoplastic lesions compared to the other categories. These results differ from a previous report in which 36 dogs and 13 cats had $7 \mathrm{MR}$ characteristic suggesting neoplasia (single lesion, regular shape, dural contact, dural tail, bone lesions, mass effect, and the presence of contrast enhancement). ${ }^{20}$ In our study population, each of those characteristics was as common to one of the other disease categories as to neoplasia. Four of those characteristics were seen with equal prevalence in all disease categories: 2 commonly (the presence of contrast enhancement, dural contact; $>50 \%$ ), and 2 uncommonly (dural tail, bone lesions; $<15 \%$ ). We found 3 characteristics that were significantly more common in inflammatory disease: meningeal enhancement, irregular lesion shape, and multifocal location (i.e., lesions involving both supratentorial and infratentorial portions of the brain). Two characteristics were found to be less common for inflammatory diseases than other categories: supratentorial location alone and the presence of a single lesion. No characteristics distinguished vascular diseases from the other two disease categories, but this might have been due to the small number of cases $(n=6)$.

Two of the characteristics which distinguished neoplasia in a previous report, ${ }^{20}$ single lesion and regular lesion shape, were equally common to neoplastic and vascular diseases in the study reported here. Indeed 19 characteristics occurred at similar frequencies in the neoplastic and vascular disease categories. Such similarity between the appearance of cerebrovascular accidents (CVA) and neoplasia seems at odds with early reports in which CVA were described as occurring most commonly in the cerebellum of dogs, having wedge shape with no mass effect and rare contrast enhancement. ${ }^{1,7}$ However a more varied appearance of CVA is described in recent reports, and the appearance depends on factors such as lesion duration, the vessel affected, and whether it is ischemic versus hemorrhagic in nature. ${ }^{6,9,26}$ In two different studies as many as $47 \%$ and $27 \%$ CVA were misdiagnosed as gliomas, while 
up to $12 \%$ of gliomas were misdiagnosed as CVA., ${ }^{9,17}$ A study also reported that $24 \%$ of CVA had mass effect and perilesional edema, similar to the appearance of gliomas. ${ }^{9}$ Although mass effect was present in almost all cases of neoplasia in our study (99\%), we also found mass effect in $65 \%$ of cases with vascular disease (one ischemic and all 3 hemorrhagic infarctions), and there was no statistical difference between the two categories. In the absence of gross hemorrhage, the presence of mass effect in CVA results from the development of cytotoxic or vasogenic edema and changes with time following the vascular event. ${ }^{27}$ With reperfusion of the ischemic tissue, MR contrast enhancement can occur later in the course of disease as brain edema and mass effect are resolving. ${ }^{6,27}$ We found a high prevalence of contrast enhancement in cases with vascular disease (64\%) which occurred at similar frequency to both other disease categories. Weak contrast enhancement was most common in vascular disease cases, similar to other studies, ${ }^{9}$ while strong enhancement was most common in our neoplastic cases. We found that a very high proportion $(90 \%)$ of histologically confirmed cases with vascular disease had a supratentorial location, similar to that of neoplasia (88\%). A high prevalence (50\%) of supratentorial CVA location in dogs has also been reported elsewhere and is likely due to lacunar (versus territorial) infarction. $^{6}$

A larger sample size and the exclusion of cats from our study population may have caused the different disease prevalence compared to the previous study. ${ }^{20}$ Intracranial neoplasias occur at different frequencies between dogs and cats. ${ }^{28-30}$ Meningioma accounts for approximately $45 \%$ of primary intracranial neoplasia and $40 \%$ of all intracranial tumors in dogs, while in cats its prevalence is estimated to range between $58 \%$ and $71 \%$ of intracranial tumors. ${ }^{28,31,32}$ In our study 19 of the 51 dogs (37.3\%) with neoplasia had meningioma, compared to 17 of 33 animals $(51.5 \%)$ in the previous report. ${ }^{20}$ Meningiomas commonly have all of the MR characteristics previously found to indicate neoplasia, ${ }^{20,21,33,34}$ while the appearance of other intracranial neoplasia can be quite different. ${ }^{3,5}$ Cats also have a much higher rate of intracranial lymphoma than dogs. ${ }^{29,32,35}$ One of 51 dogs $(2.0 \%)$ with 
neoplasia in our population had lymphoma compared to 2 of 33 animals (6.0\%) in the previous report. ${ }^{20}$ We had expected to find that a reduced prevalence of these tumor types might alter the characteristics ascribed to the neoplasia group in the previous study. However the lack of difference found between disease categories in our study appears to result from the high frequency of these MR features in one or both of the other groups, rather than a more varied appearance of neoplasia.

In this study, intra-axial lesions were more likely to be inflammatory than either of the other disease categories. By definition, meningiomas and pituitary tumors are extra-axial in origin, and their high prevalence within the neoplasia group ( $37.3 \%$ and $7.7 \%$, respectively) likely contributes to this finding. Our definition of intraventricular lesions as 'extra-axial' is common ${ }^{30,36,37}$ and is supported by the definition that extra-axial lesions arise outside of the brain parenchyma. Some references have classified intra-ventricular lesions as intra-axial while others delineate three origins (extra-axial, intra-axial, intraventricular). ${ }^{2,3,38-40}$ Designating intraventricular lesions as a distinct origin category would not have altered the prevalence of intra-axial neoplastic lesions in our analysis. It is possible that if we had defined intraventricular lesions as intra-axial in origin, there may have been no difference between the occurrence of intra-axial neoplasia and inflammatory disease. All of the intra-ventricular lesions identified in our study were neoplastic. Few reports describe inflammatory brain lesions imaged with MR as primarily affecting the ventricular system. Some inflammatory conditions are characterized by contrast enhancement of the ventricular lining or changes of T1W and T2-FLAIR signal characteristics of the CSF. ${ }^{41,42} \mathrm{~A}$ report of MR findings in dogs with inflammatory CSF did not identify lesions within the ventricles. ${ }^{43}$ Intraventricular hemorrhage has been identified with $\mathrm{MR}^{44}$ although descriptions of intraventricular masses caused by hemorrhage alone are difficult to find in the veterinary literature. It is likely that masses identified in the ventricular system without accompanying signal characteristics of hemorrhage will be neoplastic in origin. 
As in a previous report, ${ }^{20}$ standard MR signal intensity alone did not distinguish any major disease category. Heterogeneous and mixed signal intensity (T1W, T2W, T2-FLAIR) was more common to neoplasia than inflammatory lesions, though vascular lesions had similar findings to both other categories. Uniform T2W and T2-FLAIR hyperintensity was seen in almost all inflammatory lesions, but again this was not significantly different from vascular lesions. Basic MR signal characteristics have been unreliable in differentiating tumor type, tumor grade, and disease category, while reviews of descriptions from the literature reveal a great degree of both variability and overlap between diseases. ${ }^{2,4,5,8,20,21,38}$ The presence of lesion contrast enhancement was seen equally among disease categories, so contrast enhancement alone was not an indicator of neoplasia. Strong contrast enhancement was the single MR characteristic that occurred at a significantly higher frequency in neoplasia than other diseases in this study. Strong contrast enhancement is a common feature of some tumors, including meningioma, lymphoma, and choroid plexus tumors, and the degree of contrast enhancement has been associated with higher grade gliomas. ${ }^{5,10,22,25,32,38}$ Contrast enhancement has been shown to correlate well with the degree of breakdown of the blood brain barrier in gliomas, vascular proliferation, and blood vessel dilation, but was not a good predictor of histopathological processes such as necrosis in a mixed population of lesion categories. ${ }^{13,14,45}$

We were not able to show a statistical difference in the frequency of either dural tail or bone lesions between neoplasia and other diseases, based on the employed statistical model. Dural tail was observed in $9.7 \%$ of neoplasias (and in $36 \%$ of meningiomas) but was not seen in the other disease categories. As there were no observations in the other disease groups, there is no way to estimate the standard error in our statistical model. A chi-squared test comparing neoplasia to the other groups combined suggested significance $(p=0.003)$, but zero marginal totals in the other groups violated the assumptions of the test and therefore this $p$ value is likely biased, i.e., too small. This seems like a clinically relevant finding despite the limitations concerning the formal statistical comparison. The causes of dural tail may include 
direct extension of tumor, dural thickening, or vascular proliferation. ${ }^{33}$ Dural tail is a common finding in cases of meningioma, though it is not pathognomonic and previously has been found with other types of neoplasia and with inflammatory disease. ${ }^{21,33,34}$ Interobserver agreement on the presence of dural tail has been reported to be widely variable, ${ }^{19}$ which may be another limitation of relying on this finding for diagnosis. Of the dogs diagnosed with intracranial neoplasia in our study, meningeal enhancement and dural tail occurred frequently in meningioma alone (50\% and $36 \%$, respectively) and distinguished meningioma from other neoplasias. It is possible that a small sample size is responsible for a type II statistical error and that a larger sample size may have identified significant differences for dural tail and bone lesions. Nevertheless either finding in association with an extradural mass showing strong contrast enhancement is likely to indicate meningioma according to published literature. ${ }^{21,33,34}$

A recent study found that readers had higher agreement for selecting brain disease category than for individual MR features. ${ }^{19}$ Despite the lack of MR characteristic specificity and widely overlapping disease appearance, as found in the study reported here, the overall accuracy of categorizing intracranial lesions with MR is high. ${ }^{10,12,17,19}$ In a previous study, greater than $90 \%$ sensitivity (SE) and specificity (SP) for overall lesion detection with high inter-rater agreement was found. ${ }^{17}$ Some unexpected results in the previous study highlight the complexity of imaging diagnosis. Meningiomas are reported to have wellrecognized features, and indeed were diagnosed with $94.9 \%$ specificity, yet they were only identified with $59 \%$ sensitivity. ${ }^{17}$ It is also surprising that reviewer performance for identifying granulomatous meningoencephalitis (GME), a disease with a reportedly more variable appearance, ${ }^{8,46}$ was similar to that seen with meningioma ( $50 \% \mathrm{SE}, 87.9 \% \mathrm{SP}) .{ }^{17}$ An equally enlightening aspect of two recent studies is the finding that evaluator performance was generally not aided by knowledge of the patient's clinical information (breed, age, cerebrospinal fluid (CSF) results, and timing of clinical signs). ${ }^{17,19}$ In one study the addition of patient information did not significantly alter diagnosis, ${ }^{19}$ while in another the specificity of diagnosing neoplasia fell from $91.7 \%$ to $81.9 \%$ and the inter-rater agreement for diagnosing 
inflammatory disease fell from 0.564 to $0.211 .{ }^{17}$ There is an intriguing contradiction among these data. Few standard MR characteristics are specific to any disease category, and yet evaluators are making accurate diagnosis based mainly on MR appearance. One might surmise from the results of these studies that the high performance of MR for diagnosing brain diseases is based on integrating a combination of individually non-specific MR findings into patterns common to certain diseases (i.e., the combination of multiple MR characteristics is more suggestive of a disease than any of them individually). Given the possibility of similar appearance for different diseases (such as glioma and CVA), evaluators may consider additional information (such as sudden onset of clinical signs) to take precedence over some imaging findings, occasionally to their detriment. At many institutions MR exams now routinely include more advanced imaging techniques, beyond basic spin echo characteristic evaluation of tissue (T1 relaxation, T2 relaxation, and proton density) which may enhance the diagnostic accuracy of brain imaging studies. With the addition of $\mathrm{T} 2 *$-gradient echo sequences for the identification of hemorrhage breakdown products and diffusion weighted or tensor imaging (DWI, DTI) for evaluation of water diffusion, examiners are presented with more information about the nature of the tissue environment. Diffusion imaging techniques are commonly used in human medicine for the diagnosis of brain infarction, however even these techniques lack perfect accuracy. One study found that quantitative ADC performed at a single time point was also unable to differentiate between glial tumors, CVA, and GME in dogs. ${ }^{47}$ It is likely that the fundamental task of MR interpretation will continue to involve complex pattern recognition in decision making.

One limitation of the study is the requirement for histopathologically confirmed brain disease. This selection criterion may have contributed to the low number of cases with vascular disease, which often has a more favorable prognosis and is not commonly biopsied when presumptively diagnosed. Since this was a retrospective study, we were not able to standardize MR equipment or protocol. Our inclusion criteria limited cases to those studies performed at $1 T$ or higher field strength to limit variability caused 
by vastly different image quality. We also withheld additional MR sequences and image planes when available so that each case presented the examiner with only a uniform set of sequences. Still, variability in image quality, resolution, and field strength may have affected lesion characteristic appearance and is a limitation of this study. The employed statistical model was able to adjust for the repeated observations on each dog by the incorporation of a random effect term. However, there is no closed form for maximizing the likelihood function and the small number of observations per dog leads to instability in estimating the random effect. Also, complete separation of groups, such as observed for dural tail, will cause problems with the estimation of the random effect limiting the inferences that are possible. An alternative approach to using this statistical model would have been to obtain consensus information for all readers and then perform logistic regression without inclusion of a random effect.

In conclusion, we were able to validate only strong contrast enhancement as a finding more common to neoplasia than to inflammatory or vascular diseases. We did identify meningeal enhancement, irregular lesion shape, and multifocal location (both supratentorial and infratentorial) as MR findings which were more common to inflammatory diseases alone. The characteristics found in vascular diseases were similar to both other disease categories and were most similar to neoplasia. The high performance of MR for diagnosing intracranial diseases reported elsewhere might be due to evaluator recognition of combinations of MR characteristics versus relying on the specificity of any characteristic alone. 


\section{REFERENCES}

1. Thomas WB, Sorjonen DC, Scheuler RO, et al. Magnetic resonance imaging of brain infarction in seven dogs. Vet Radiol Ultrasound 1996;37:345-350.

2. Thomas WB, Wheeler SJ, Kramer R, et al. Magnetic Resonance Imaging Features of Primary Brain Tumors in Dogs. Vet Radiol Ultrasound 1996;37:20-27.

3. Wisner ER, Dickinson PJ, Higgins RJ. Magnetic resonance imaging features of canine intracranial neoplasia. Vet Radiol Ultrasound 2011;52:S52-S61.

4. Young BD, Levine JM, Fosgate GT, et al. Magnetic resonance imaging characteristics of necrotizing meningoencephalitis in Pug dogs. J Vet Intern Med 2009;23:527-535.

5. Young $\mathrm{BD}$, Levine JM, Porter BF, et al. Magnetic resonance imaging features of intracranial astrocytomas and oligodendrogliomas in dogs. Vet Radiol Ultrasound 2011;52:132-141.

6. Garosi L, McConnell JF, Platt SR, et al. Clinical and Topographic Magnetic Resonance Characteristics of Suspected Brain Infarction in 40 Dogs. J Vet Intern Med 2006;20:311-321.

7. McConnell JF, Garosi L, Platt SR. Magnetic resonance imaging findings of presumed cerebellar cerebrovascular accident in twelve dogs. Vet Radiol Ultrasound 2005;46:1-10.

8. Cherubini GB, Platt SR, Anderson TJ, et al. Characteristics of magnetic resonance images of granulomatous meningoencephalomyelitis in 11 dogs. Vet Rec 2006;159:110-115.

9. Cervera $\mathrm{V}$, Mai W, Vite $\mathrm{CH}$, et al. Comparative magnetic resonance imaging findings between gliomas and presumed cerebrovascular accidents in dogs. Vet Radiol Ultrasound 2011;52:33-40.

10. Ródenas S, Pumarola M, Gaitero L, et al. Magnetic resonance imaging findings in 40 dogs with histologically confirmed intracranial tumours. The Veterinary Journal 2011;187:85-91.

11. Vite $\mathrm{CH}$, Cross JR. Correlating magnetic resonance findings with neuropathology and clinical signs in dogs and cats. Vet Radiol Ultrasound 2011;52:S23-S31.

12. Polizopoulou ZS, Koutinas AF, Souftas VD, et al. Diagnostic correlation of CT-MRI and histopathology in 10 dogs with brain neoplasms. J Vet Med 2004;51:226-231.

13. Brunner Singh J, Oevermann A, Lang J, et al. Contrast media enhancement of intracranial lesions in magnetic resonance imaging does not reflect histopathologic findings consistently. Vet Radiol Ultrasound 2011;52:619-626.

14. Pronin IN, Holodny AI, Petraikin AV. MRI of high-grade glial tumors: correlation between the degree of contrast enhancement and the volume of surrounding edema. Neuroradiology 1997;39:348-350.

15. van der Knaap MS, van der Grond J, Luyten PR, et al. $1 \mathrm{H}$ and 31P magnetic resonance spectroscopy of the brain in degenerative cerebral disorders. Ann Neurol 1992;31:202-211.

16. Julia-Sape $M$, Acosta D, Majos $C$, et al. Comparison between neuroimaging classifications and histopathological diagnoses using an international multicenter brain tumor magnetic resonance imaging database. J Neurosurg 2006;105:6-14.

17. Wolff $C A$, Holmes SP, Young BD, et al. Magnetic resonance imaging for the differentiation of neoplastic, inflammatory, and cerebrovascular brain disease in dogs. J Vet Intern Med 2012;26:589-597.

18. Hagen T, Nieder C, Moringlane JR, et al. [Correlation of preoperative neuroradiologic with postoperative histologic diagnosis in pathological intracranial processes]. Der Radiologe 1995;35:808815.

19. Leclerc M-K, d'Anjou M-A, Blond L, et al. Interobserver agreement and diagnostic accuracy of brain magnetic resonance imaging in dogs. J Am Vet Med Assoc 2013;242:1688-1695.

20. Cherubini GB, Mantis $P$, Martinez TA, et al. Utility of magnetic resonance imaging for distinguishing neoplastic from non-neoplastic brain lesions in dogs and cats. Vet Radiol Ultrasound 2005;46:384-387.

21. Sturges BK. Magnetic resonance imaging and histological classification of intracranial meningiomas in 112 dogs. J Vet Intern Med 2008;22:586. 
22. Palus $V$, Volk HA, Lamb CR, et al. MRI features of CNS lymphoma in dogs and cats. Vet Radiol Ultrasound 2012;53:44-49.

23. Koch MW, Sánchez MD, Long S. Multifocal oligodendroglioma in three dogs. J Am Anim Hosp Assoc 2011;47:e77-e85.

24. Lipsitz D, Higgins RJ, Kortz GD, et al. Glioblastoma multiforme: clinical findings, magnetic resonance imaging, and pathology in five dogs. Vet Pathol 2003;40:659-669.

25. Westworth DR, Dickinson PJ, Vernau W, et al. Choroid plexus tumors in 56 dogs (1985-2007). J Vet Intern Med 2008;22:1157-1165.

26. Lowrie M, De Risio L, Dennis R, et al. Concurrent medical conditions and long-term outcome in dogs with nontraumatic intracranial hemorrhage. Vet Radiol Ultrasound 2012;53:381-388.

27. Marks MP. Cerebral ischemia and infarction. In: Atlas SW, ed. Magnetic resonance imaging of the brain and spine, 4 ed. Philidelphia, PA: Wolters Kluwer, Lippincott Williams \& Wilkins; 2009:772-825.

28. Hayes HM, Priester WA, Jr., Pendergrass TW. Occurrence of nervous-tissue tumors in cattle, horses, cats and dogs. Int J Cancer 1975;15:39-47.

29. Troxel MT, Vite $\mathrm{CH}$, van Winkle TJ, et al. Feline Intracranial Neoplasia: Retrospective Review of 160 Cases (1985-2001). J Vet Intern Med 2003;17:850-859.

30. Troxel $\mathrm{MT}$, Vite $\mathrm{CH}$, Massicotte $\mathrm{C}$, et al. Magnetic Resonance Imaging Features of Feline Intracranial Neoplasia: Retrospective Analysis of 46 Cats. J Vet Intern Med 2004;18:176-189.

31. Heidner GL, Kornegay JN, Page RL, et al. Analysis of survival in a retrospective study of 86 dogs with brain tumors. J Vet Intern Med 1991;5:219-226.

32. Snyder JM, Shofer FS, Van Winkle TJ, et al. Canine intracranial primary neoplasia: 173 cases (19862003). J Vet Intern Med 2006;20:669-675.

33. Graham JP, Newell SM, Voges AK, et al. The dural tail sign in the diagnosis of meningiomas. Vet Radiol Ultrasound 1998;39:297-302.

34. Hathcock JT. Low field magnetic resonance imaging characteristics of cranial vault meningiomas in 13 dogs. Vet Radiol Ultrasound 1996;37:257-263.

35. Snyder JM, Lipitz L, Skorupski KA, et al. Secondary Intracranial Neoplasia in the Dog: 177 Cases (1986-2003). J Vet Intern Med 2008;22:172-177.

36. Kraft SL, Gavin PR. Intracranial neoplasia. Clin Tech Small Anim Pract 1999;14:112-123.

37. Ohlerth S, Scharf G. Computed tomography in small animals - Basic principles and state of the art applications. The Veterinary Journal 2007;173:254-271.

38. Kraft SL, Gavin PR, DeHaan C, et al. Retrospective review of 50 canine intracranial tumors evaluated by magnetic resonance imaging. J Vet Intern Med 1997;11:218-225.

39. Vural SA, Besalti O, Ilhan F, et al. Ventricular ependymoma in a German Shepherd dog. The Veterinary Journal 2006;172:185-187.

40. Platt SR. Canine central nervous system inflammatory diseases: a common quandary? J Small Anim Pract 2006;47:571-572.

41. Seiler G, Cizinauskas S, Scheidegger J, et al. Low-field magnetic resonance imaging of a pyocephalus and a suspected brain abscess in a German shepherd dog. Vet Radiol Ultrasound 2001;42:417-422.

42. Negrin A, Lamb CR, Cappello R, et al. Results of magnetic resonance imaging in 14 cats with meningoencephalitis. J Feline Med Surg 2007;9:109-116.

43. Lamb CR, Croson PJ, Cappello R, et al. Magnetic resonance imaging findings in 25 dogs with inflammatory cerebrospinal fluid. Vet Radiol Ultrasound 2005;46:17-22.

44. Garosi LS, Platt SR, McConnell JF, et al. Intracranial haemorrhage associated with Angiostrongylus vasorum infection in three dogs. J Small Anim Pract 2005;46:93-99.

45. Holodny Al, Nusbaum AO, Festa S, et al. Correlation between the degree of contrast enhancement and the volume of peritumoral edema in meningiomas and malignant gliomas. Neuroradiology 1999;41:820-825. 
46. Lobetti RG, Pearson J. Magnetic resonance imaging in the diagnosis of focal granulomatous meningoencephalitis in two dogs. Vet Radiol Ultrasound 1996;37:424-427.

47. Sutherland-Smith J, King R, Faissler D, et al. Magnetic resonance imaging apparent diffusion coefficients for histologically confirmed intracranial lesions in dogs. Vet Radiol Ultrasound 2011;52:142148. 
Table 1. High-field magnetic resonance (MR) findings of histologically confirmed neoplastic, inflammatory, and vascular brain diseases in dogs.

\begin{tabular}{|c|c|c|c|c|}
\hline & $\begin{array}{c}\text { Neoplasia } \\
\qquad(n=52)\end{array}$ & $\begin{array}{l}\text { Inflammatory } \\
(\mathrm{n}=18)\end{array}$ & $\begin{array}{c}\text { Vascular } \\
(n=6)\end{array}$ & \\
\hline MR finding & $\begin{array}{l}\text { Proportion } \\
\text { (95\% CI) }\end{array}$ & $\begin{array}{l}\text { Proportion } \\
\text { (95\% CI) }\end{array}$ & $\begin{array}{l}\text { Proportion } \\
\text { (95\% CI) }\end{array}$ & $P$ value \\
\hline T1W heterogeneous & $0.71^{\mathrm{a}}(0.59,0.80)$ & $0.25^{b}(0.12,0.45)$ & $0.47^{\mathrm{a}, \mathrm{b}}(0.15,0.81)$ & 0.001 \\
\hline T2W heterogeneous & $0.89^{\mathrm{a}}(0.81,0.94)$ & $0.54^{\mathrm{b}}(0.33,0.74)$ & $0.62^{\mathrm{a}, \mathrm{b}}(0.24,0.89)$ & 0.002 \\
\hline T1W isointensity & $0.08^{\mathrm{a}}(0.03,0.16)$ & $0.43^{\mathrm{b}}(0.21,0.69)$ & $0.29^{\mathrm{a}, \mathrm{b}}(0.05,0.76)$ & 0.005 \\
\hline T1W mixed intensity & $0.48^{\mathrm{a}}(0.36,0.61)$ & $0.18^{\mathrm{b}}(0.08,0.36)$ & $0.39^{\mathrm{a}, \mathrm{b}}(0.12,0.76)$ & 0.030 \\
\hline T2W hypo intensity & $0.03(0.01,0.07)$ & $0.03(0.01,0.13)$ & $0.13(0.03,0.41)$ & 0.167 \\
\hline T2W hyperintensity & $0.39^{\mathrm{a}}(0.28,0.51)$ & $0.95^{\mathrm{b}}(0.84,0.99)$ & $0.55^{\mathrm{a}, \mathrm{b}}(0.22,0.85)$ & $<0.001$ \\
\hline T2W mixed intensity & $0.59^{\mathrm{a}}(0.47,0.70)$ & $0.05^{\mathrm{b}}(0.01,0.16)$ & $0.31^{\mathrm{a}, \mathrm{b}}(0.09,0.67)$ & $<0.001$ \\
\hline T2 FLAIR heterogeneous & $0.89^{\mathrm{a}}(0.81,0.94)$ & $0.58^{\mathrm{b}}(0.36,0.78)$ & $0.51^{\mathrm{a}, \mathrm{b}}(0.17,0.84)$ & 0.003 \\
\hline T2 FLAIR hyperintensity & $0.23^{\mathrm{a}}(0.14,0.35)$ & $0.96^{\mathrm{b}}(0.84,0.99)$ & $0.56^{\mathrm{a}, \mathrm{b}}(0.20,0.87)$ & $<0.001$ \\
\hline T2 FLAIR mixed intensity & $0.71^{\mathrm{a}}(0.59,0.81)$ & $0.05^{\mathrm{b}}(0.01,0.16)$ & $0.37^{\mathrm{a}, \mathrm{b}}(0.11,0.74)$ & $<0.001$ \\
\hline Contrast enhancement & $0.93(0.86,0.97)$ & $0.87(0.68,0.95)$ & $0.64(0.23,0.91)$ & 0.108 \\
\hline Weak enhancement & $0.21^{\mathrm{a}}(0.12,0.33)$ & $0.52^{\mathrm{a}}(0.28,0.75)$ & $0.56^{\mathrm{a}}(0.18,0.89)$ & 0.032 \\
\hline Strong enhancement & $0.70^{\mathrm{a}}(0.53,0.83)$ & $0.25^{\mathrm{b}}(0.09,0.53)$ & $0.05^{\mathrm{b}}(0.00,0.47)$ & 0.003 \\
\hline Heterogeneous contrast & $0.50(0.38,0.62)$ & $0.72(0.53,0.86)$ & $0.34(0.10,0.71)$ & 0.082 \\
\hline Intra-axial origin & $0.37^{\mathrm{a}}(0.22,0.55)$ & $0.93^{\mathrm{b}}(0.75,0.98)$ & $1.00^{\mathrm{b}}(0.00,1.00)$ & 0.002 \\
\hline Supratentorial location & $0.88^{\mathrm{a}}(0.77,0.94)$ & $0.33^{\mathrm{b}}(0.14,0.61)$ & $0.90^{\mathrm{a}}(0.43,0.99)$ & 0.001 \\
\hline
\end{tabular}




\begin{tabular}{lcccc} 
Multifocal location & $0.05^{\mathrm{a}}(0.02,0.11)$ & $0.41^{\mathrm{b}}(0.21,0.65)$ & $0.06^{\mathrm{a}}(0.01,0.44)$ & $<0.001$ \\
Mixed lesion distribution & $0.91(0.86,0.95)$ & $0.80(0.67,0.88)$ & $0.80(0.53,0.93)$ & 0.063 \\
Meningeal-dural contact & $0.87(0.76,0.93)$ & $0.70(0.43,0.88)$ & $0.58(0.17,0.91)$ & 0.149 \\
Irregular lesion shape & $0.50^{\mathrm{a}}(0.41,0.60)$ & $0.96^{\mathrm{b}}(0.86,0.99)$ & $0.47^{\mathrm{a}}(0.21,0.74)$ & $<0.001$ \\
Single lesion & $0.88^{\mathrm{a}}(0.77,0.94)$ & $0.17^{\mathrm{b}}(0.05,0.42)$ & $0.80^{\mathrm{a}}(0.29,0.98)$ & $<0.001$ \\
Defined margins & $0.79^{\mathrm{a}}(0.67,0.88)$ & $0.06^{\mathrm{b}}(0.02,0.19)$ & $0.35^{\mathrm{a}, \mathrm{b}}(0.09,0.76)$ & $<0.001$ \\
Mass effect & $0.99^{\mathrm{a}}(0.94,1.00)$ & $0.55^{\mathrm{b}}(0.31,0.78)$ & $0.65^{\mathrm{a}, \mathrm{b}}(0.22,0.93)$ & $<0.001$ \\
Meningeal enhancement & $0.22^{\mathrm{a}}(0.14,0.33)$ & $0.59^{\mathrm{b}}(0.37,0.77)$ & $0.06^{\mathrm{a}}(0.01,0.41)$ & 0.003 \\
Dural tail & $0.10(0.05,0.19)$ & $0.00(0.00,1.00)$ & $0.00(0.00,1.00)$ & $0.003^{*}$ \\
\hline
\end{tabular}

Proportions without superscripts in common are significantly different

$\mathrm{CI}=$ confidence interval

*Mantel-Haenszel chi-square comparing the neoplasia group to a combined inflammatory and vascular group while stratifying by reader 
Table 2. Multivariable logistic regression for the prediction of histologically confirmed neoplastic lesions in dogs using high-field magnetic resonance (MR) findings.

\begin{tabular}{lccc}
\hline \multicolumn{1}{c}{ MR finding } & $\begin{array}{c}\text { Parameter } \\
\text { estimate }(\hat{\beta})\end{array}$ & $\begin{array}{c}\text { P value } \\
\text { (t statistic) }\end{array}$ & $\begin{array}{c}\text { Odds ratio } \\
\mathbf{( 9 5 \% ~ C I ) ~}\end{array}$ \\
\hline Defined margins & 2.473 & 0.002 & $11.9(2.54,55.3)$ \\
T2FLAIR mixed intensity & 2.015 & 0.013 & $7.50(1.55,36.4)$ \\
Extra-axial origin & 2.658 & 0.006 & $14.3(2.17,93.9)$ \\
\hline CI = confidence interval. & & &
\end{tabular}


Table 3. High-field magnetic resonance (MR) findings of histologically confirmed neoplastic brain diseases in dogs.

\begin{tabular}{|c|c|c|c|c|}
\hline & $\begin{array}{l}\text { Meningioma } \\
\qquad(\mathrm{n}=19)\end{array}$ & $\begin{array}{l}\text { Glioma } \\
(n=15)\end{array}$ & $\begin{array}{l}\text { Other } \\
(n=18)\end{array}$ & \\
\hline MR finding & $\begin{array}{l}\text { Proportion } \\
\text { (95\% CI) }\end{array}$ & $\begin{array}{l}\text { Proportion } \\
\text { (95\% CI) }\end{array}$ & $\begin{array}{l}\text { Proportion } \\
(95 \% \text { CI) }\end{array}$ & P value \\
\hline T1W hypo intensity & $0.32^{\mathrm{a}}(0.16,0.53)$ & $0.66^{\mathrm{b}}(0.44,0.83)$ & $0.13^{\mathrm{a}}(0.05,0.30)$ & 0.002 \\
\hline T1W mixed intensity & $0.41(0.22,0.62)$ & $0.34(0.17,0.57)$ & $0.69(0.48,0.84)$ & 0.056 \\
\hline T2 FLAIR heterogeneous & $0.78(0.53,0.92)$ & $0.94(0.76,0.99)$ & $0.97(0.83,1.00)$ & 0.105 \\
\hline Contrast enhancement & $0.94(0.78,0.99)$ & $0.85(0.62,0.96)$ & $0.98(0.86,1.00)$ & 0.194 \\
\hline Weak enhancement & $0.10^{\mathrm{a}}(0.03,0.27)$ & $0.43^{\mathrm{a}}(0.21,0.68)$ & $0.17^{\mathrm{a}}(0.06,0.38)$ & 0.050 \\
\hline Strong enhancement & $0.84^{\mathrm{a}}(0.62,0.94)$ & $0.32^{\mathrm{b}}(0.13,0.60)$ & $0.82^{\mathrm{a}}(0.58,0.93)$ & 0.007 \\
\hline Homogeneous contrast & $0.39^{\mathrm{a}}(0.22,0.59)$ & $0.04^{\mathrm{b}}(0.01,0.17)$ & $0.32^{\mathrm{a}}(0.17,0.52)$ & 0.012 \\
\hline Heterogeneous contrast & $0.50(0.30,0.70)$ & $0.33(0.16,0.56)$ & $0.64(0.43,0.81)$ & 0.139 \\
\hline Rim contrast pattern & $0.02^{\mathrm{a}}(0.00,0.14)$ & $0.36^{\mathrm{a}}(0.14,0.67)$ & $0.02^{\mathrm{a}}(0.00,0.14)$ & 0.004 \\
\hline Intra-axial origin & $0.12^{\mathrm{a}}(0.05,0.29)$ & $0.98^{\mathrm{b}}(0.85,1.00)$ & $0.13^{\mathrm{a}}(0.05,0.31)$ & $<0.001$ \\
\hline Multifocal lesion & $0.02(0.00,0.14)$ & $0.02(0.00,0.16)$ & $0.11(0.04,0.31)$ & 0.165 \\
\hline Meningeal-dural contact & $0.97(0.84,1.00)$ & $0.81(0.58,0.93)$ & $0.78(0.54,0.91)$ & 0.104 \\
\hline Meningeal enhancement & $0.50^{\mathrm{a}}(0.32,0.68)$ & $0.02^{\mathrm{b}}(0.00,0.15)$ & $0.19^{\mathrm{c}}(0.09,0.35)$ & 0.001 \\
\hline Dural tail & $0.36^{\mathrm{a}}(0.17,0.59)$ & $0.00^{\mathrm{b}}(0.00,1.00)$ & $0.02^{\mathrm{b}}(0.00,0.13)$ & 0.019 \\
\hline
\end{tabular}

Proportions without superscripts in common are significantly different 